\title{
Enhanced Light Extraction by Photonic Quasi-Crystals in GaN Blue LEDs
}

\author{
Philip A. Shields, Martin D. B. Charlton, Tom Lee, Majd E. Zoorob, Duncan W. E. Allsopp, Member, IEEE, \\ and Wang N. Wang, Member, IEEE
}

\begin{abstract}
The far-field profile of photonic quasi-crystal patterned and unpatterned LEDs, fabricated from commercial epitaxial substrates by electron beam lithography, has been measured prior to lapping and dicing. Emission enhancements reach a maximum of $62 \%$, and are strongly dependent on the filling factor. Qualitative agreement is achieved between 2-D finite-difference time-domain calculations and the experimental data.
\end{abstract}

Index Terms-Electroluminescence, extraction, LEDs, photonic crystals.

\section{INTRODUCTION}

$\mathbf{T}$ HE LAST few years has seen reports of the use of photonic crystals to increase the extraction efficiency of LEDs by coupling the traditionally trapped guided modes in a planar semiconductor structure into radiative modes [1]. Initially, work focused on the theoretical possibilities that photonic crystals can alter the spontaneous emission within an LED via the Purcell effect [2], or used bespoke structures or excitation schemes to demonstrate the principles [3]-[5]. Subsequently, there was a shift toward photonic crystal integration in more commercial LED devices [6].

In order to increase the strength of the interaction between the guided modes and the photonic crystal, the use of a buried photonic crystal has been reported [7], [8]. However, this approach requires a second epitaxial growth process for the regrowth of the capping material. The growth is more challenging, and the intermediate processing step will introduce contamination close to the critical active layers.

The approach taken in this paper is to introduce the periodic structure in the top layer of the high-index GaN waveguide. Since the photonic crystal is separated from the active region, this approach introduces only a small perturbation to the trapped optical modes. Therefore, it is not the photonic bandgap that is being exploited but the coupling of trapped modes with modes that can escape from the material. This simpler process enabled the trends over a relatively wide range of the photonic crystal

Manuscript received December 1, 2008; revised February 2, 2009. First published May 19, 2009; current version published August 5, 2009. This work was supported by the U.K. Technology Strategy Board.

P. A. Shields, D. W. E. Allsopp, and W. N. Wang are with the University of Bath, Bath BA2 7AY, U.K. (e-mail: p.shields@bath.ac.uk).

M. D. B. Charlton is with Mesophotonics Ltd., Southampton SO16 7NP, U.K., and also with the University of Southampton, Southampton SO17 1BJ, U.K.

T. Lee and M. E. Zoorob were with Mesophotonics Ltd., Southampton SO16 7NP, U.K. They are now with PhotonStar LED Ltd., Southampton SO16 7NP, U.K.

Color versions of one or more of the figures in this paper are available online at http://ieeexplore.ieee.org.

Digital Object Identifier 10.1109/JSTQE.2009.2016674 design parameters to be studied using readily available commercial epitaxy, but with the drawback that the layer structure may not be the optimum for strong-mode perturbation.

One of the disadvantages of using square or triangular symmetry photonic crystals to improve LED extraction is the azimuthal anisotropy of the light emission that is undesirable in lighting applications [9]. An alternative is to use photonic quasicrystals (PQCs) that have higher rotational symmetries leading to less azimuthal emission dependency [10]-[12]. These quasicrystals have long-range order but no short-range translational order.

\section{EXPERIMENTAL DETAILS}

Standard unroughened GaN-based LED wafers on sapphire substrates were sourced from commercial suppliers. The surface was patterned with electron-beam lithography (EBL) before the resist pattern (ZEP 520, Zeon Corporation) was transferred via a $\mathrm{SiO}_{2}$ hard mask to the upper 200-nm-thick p-GaN layer by $\mathrm{Cl}_{2}$ /Ar plasma etching. The patterned wafer was then processed by conventional lithography into $270 \times 270 \mu \mathrm{m}$ LED chips using 5:5 nm Ni/Au transparent p-contacts.

The PQC patterns were based on square-triangular tiling [10] with three different pitches: 450, 550, and $750 \mathrm{~nm}$. Initially, we aimed for hole diameters of half the pitch, which corresponds to a filling factor or air fraction of $\sim 0.22$. In practice, three different beam doses were used in order to achieve three different hole sizes around the design filling factor. Also, each element was written as a square by the EBL system rather than a circle, since this considerably increased the writing speed allowing many more LEDs to be patterned in one run. This enabled a sufficient number of devices to be fabricated for systematic trends to be studied without drift in the EBL system being a factor. Thus, the experimental realizations only approximate to the idealized structures.

Fig. 1 shows a SEM image of one of the photonic crystal chips (PCLED) with a representative area of the quasi-photonic crystal structure defined in its top surface. The depth of the etched holes was measured to be $110 \mathrm{~nm}$ by atomic force microscope (AFM). The inset at highest magnification indicates the degree of distortion that occurred to the elements that were written as individual squares.

The SEM observations of the final LEDs reveal that the fabricated patterns differ from that intended in the following different ways: the positional accuracy of the element, its size, and its circularity. Measurements of the geometric parameters of the photonic crystal structures were obtained from SEM images in order to assess the departure from that originally intended [13] 


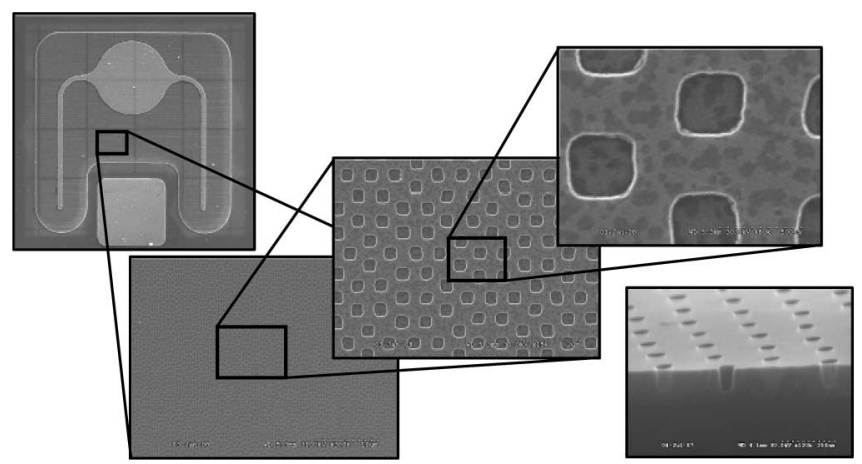

Fig. 1. SEM images of a 750-nm-pitch PCLED along with the cross section from a test sample showing the hole profile.

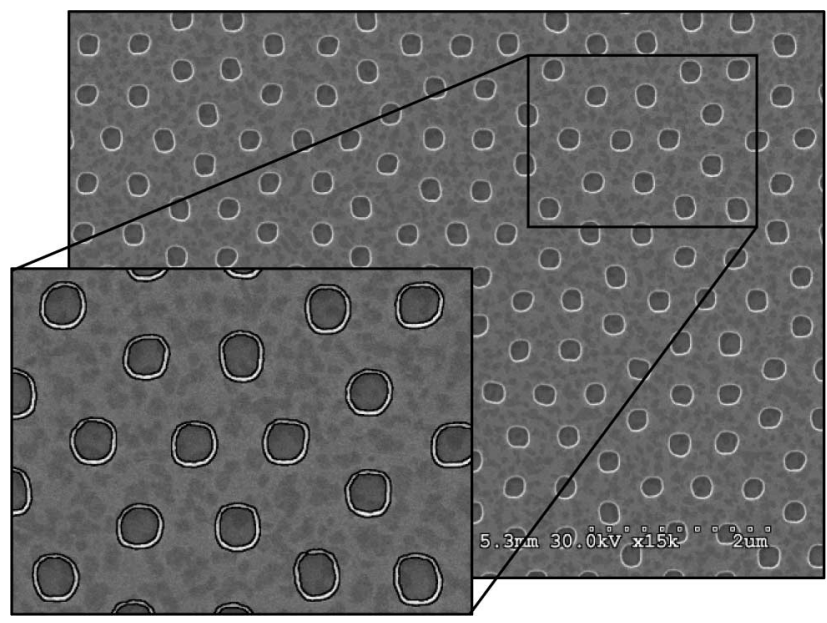

Fig. 2. Example of the SEM image analysis that has been used to determine the fidelity of the photonic crystal. The inset is annotated with the lateral extent of the top and bottoms of the etched holes.

TABLE I

PCLED PARAMETERS DEDUCED FROM THE SEM AND AFM ANALYSIS THAT DESCRIBE THE PATTERN FIDELITY

\begin{tabular}{ccccc}
\hline & Intended & \multicolumn{3}{c}{ Actual } \\
& & $450 \mathrm{~nm}$ & $550 \mathrm{~nm}$ & $750 \mathrm{~nm}$ \\
Air fraction & 0.22 & 0.13 & 0.13 & 0.24 \\
& & 0.17 & 0.16 & 0.27 \\
Positional error & $0 \%$ & 0.21 & 0.18 & 0.30 \\
Diameter error & $0 \%$ & $5 \%$ & $4 \%$ & $5 \%$ \\
Sidewall angle & $90^{\circ}$ & $77^{\circ}$ & $77^{\circ}$ & $77^{\circ}$ \\
Circularity & $100 \%$ & $87 \%$ & $87 \%$ & $74 \%$ \\
\hline
\end{tabular}

The SEM images were analyzed to determine the nearest neighbor distances, the distribution of hole sizes, the hole circularity, and, in combination with AFM measurements of the hole depth, the sidewall angle. Fig. 2 shows an example of such an SEM image with the inset additionally annotated with the lateral extent of the top and bottoms of the holes. Table I lists the parameters deduced from the SEM and AFM analysis that describe the pattern fidelity.

For characterization, an optical fiber mounted on a goniometer was used to measure the far-field light distribution of the undiced devices. Control samples with no surface patterning were interspersed between every patterned LED to minimize the influence on the results of growth and fabrication variations across the wafer fragment. Since the wafer was not diced into chips, these results do not include the effects of emission through the cleaved chip sidewalls. This forms a significant contribution to the total output of diced chips as reported in [14]. Nevertheless, without singulation, comparisons are easier since all that is required for comparing extraction enhancements from differently patterned chips is a simple translation of the wafer by the chip pitch.

\section{RESULTS}

Fig. 3 shows the angular electroluminescence from an unpatterned control LED (upper left) and a patterned PQC LED (upper right) as a function of zenith angle and wavelength. The measurements were for TE polarization. Numerous peaks can be observed in the control sample. Those that shift angle with wavelength correspond to Fabry-Perot (FP) fringes that arise from sandwiching the $\sim 4-\mu$ m high-index GaN layer between the lower index sapphire substrate and air. Two further peaks at $\pm 79^{\circ}$ that are independent of wavelength correspond to lateral emission from the etched mesa sidewalls.

The FP modes correspond to modes that lie within the normal material extraction cone, and would be subject to Fresnel reflection at the $\mathrm{GaN}$-air interface. This provides limited recycling of photons within the structure, and hence, a very weak interaction with the photonic crystal surface patterning. Nonetheless, for the patterned sample, these broad FP fringes are almost completely broken down, as shown in the lower two graphs of Fig. 3 . This provides information about the level of interaction between the relatively shallow photonic crystal and the weakly confined FP modes. These effects have been examined in detail for thin waveguide structures in [15]. Energy previously associated with these weakly confined modes has become redistributed in the far field. In Fig. 3 (lower graphs), we see that the two FP modes closest to the critical angle at $50^{\circ}$ and $65^{\circ}$ are efficiently smeared out, and only the two remaining FP modes at $0^{\circ}$ and $30^{\circ}$ survive but are less pronounced. We would expect there to be a relationship between the number of FP modes extracted by the PC and the etch depth of the holes. The data indicate that the photonic crystal, in this case, of depth $110 \mathrm{~nm}$ interacts with the higher order FP modes but is not deep enough to interact strongly with the remaining lower order FP modes. An identical behavior is observed for TM-like modes.

The analysis of FP modal behavior provides an insight into the relationships and level of interaction between the photonic crystal and light extracted from the structure but does not tell us anything more about the interaction of the photonic crystal with guided modes that occur as a result of total internal reflection.

Looking more closely at Fig. 3 (top right), inclined lines are observed from the Bragg scattering of the confined optical modes by the photonic crystal. These can be seen more clearly by taking a cross section through the contour plots at $454 \mathrm{~nm}$, as shown in the lower two graphs of Fig. 3. The fine structure can be seen more clearly along with the emission enhancement with the reminder that the jagged line for the PCLED is not 

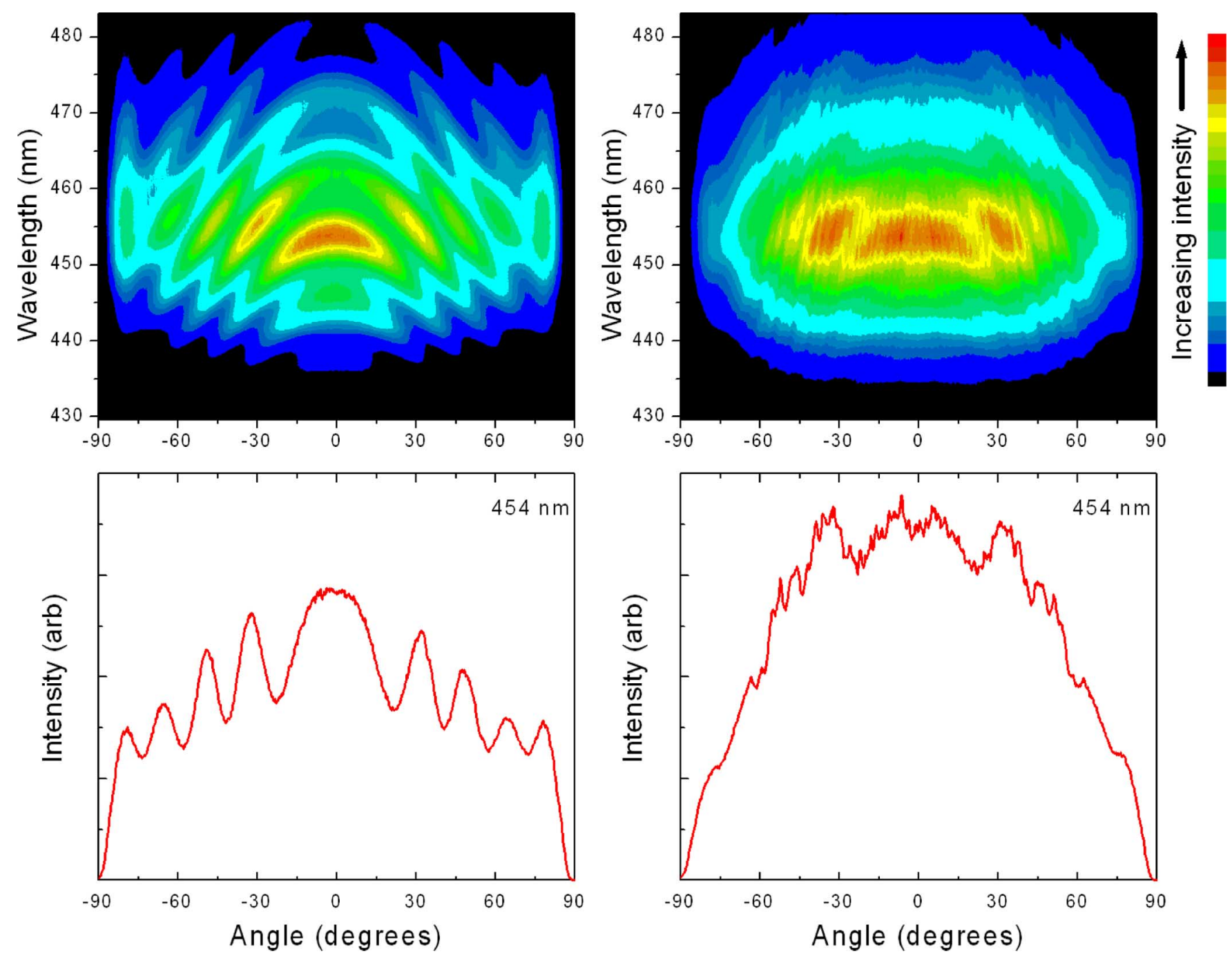

Fig. 3. Angular electroluminescence plots for TE-polarized light for a control LED (left) and a 750-nm-pitch PCLED (right). The intensity versus wavelength is shown in the form of contour plots for the full emission band (above) and as line plots for a single wavelength of $454 \mathrm{~nm}$ (below). The centre of the $x$-axis corresponds to the surface normal.

caused by noise but rather by the numerous Bragg scattered modes. This fine structure confirms that the photonic crystal does not merely act as a source of inclined surface normals akin to surface roughening. Rather, it has a significant interaction with the trapped guided modes as well. This is further confirmed by the strong reduction in the mesa sidewall emission at $\pm 79^{\circ}$ for the photonic crystal sample. As expected, it shows that with the increased light extraction through the top patterned surface, there is a simultaneous reduction of optical energy in the guided modes.

Integrating over the wide $\sim 40 \mathrm{~nm}$ emission band leads to all the F-P and the Bragg features being smeared out. For the unpatterned control samples, an almost perfect Lambertian farfield profile is recovered, thus confirming the validity of the experimental setup. Within a $\pm 50^{\circ}$ cone, this emission was Lambertian to within $\pm 2 \%$. Outside this range, small peaks representing $5 \%$ of the total light output were observed due to emission through the mesa sidewalls.

Between successive measurements, the wafer was translated by the chip pitch in order to allow direct comparison of the light output intensity of patterned and unpatterned devices. The electrical characteristics of the PCLEDs and the control LEDs were very similar with a forward voltage of $3.2 \mathrm{~V}$ at $20 \mathrm{~mA}$ drive current.

Fig. 4(a) shows the measured far-field profile of both the control LEDs and the PCLEDs for the three different pitches. The EBL dose was the same $\left(150 \mu \mathrm{C} / \mathrm{cm}^{2}\right)$ for the three PQC structures considered, and corresponds to the highest dose used in this paper. The total emission intensity enhancement from the top surface for the best chips for each pitch was estimated by integrating the far-field profile over the extraction cone, whilst assuming that there is no azimuthal dependence on the far-field profile.

In order to confirm this assumption, a second experiment was performed at a fixed zenith angle of $30^{\circ}$ for a sample with a 735-nm PQC etched in the surface but without metal contacts or $\mathrm{Ni}-\mathrm{Au}$ conducting layer. Luminescence that was excited by a $\mathrm{He}-\mathrm{Cd}$ laser at $325 \mathrm{~nm}$ was collected by the same experimental setup as before for azimuthal angles between $0^{\circ}-180^{\circ}$. The results are shown in Fig. 5. The contour plot shows intensity 


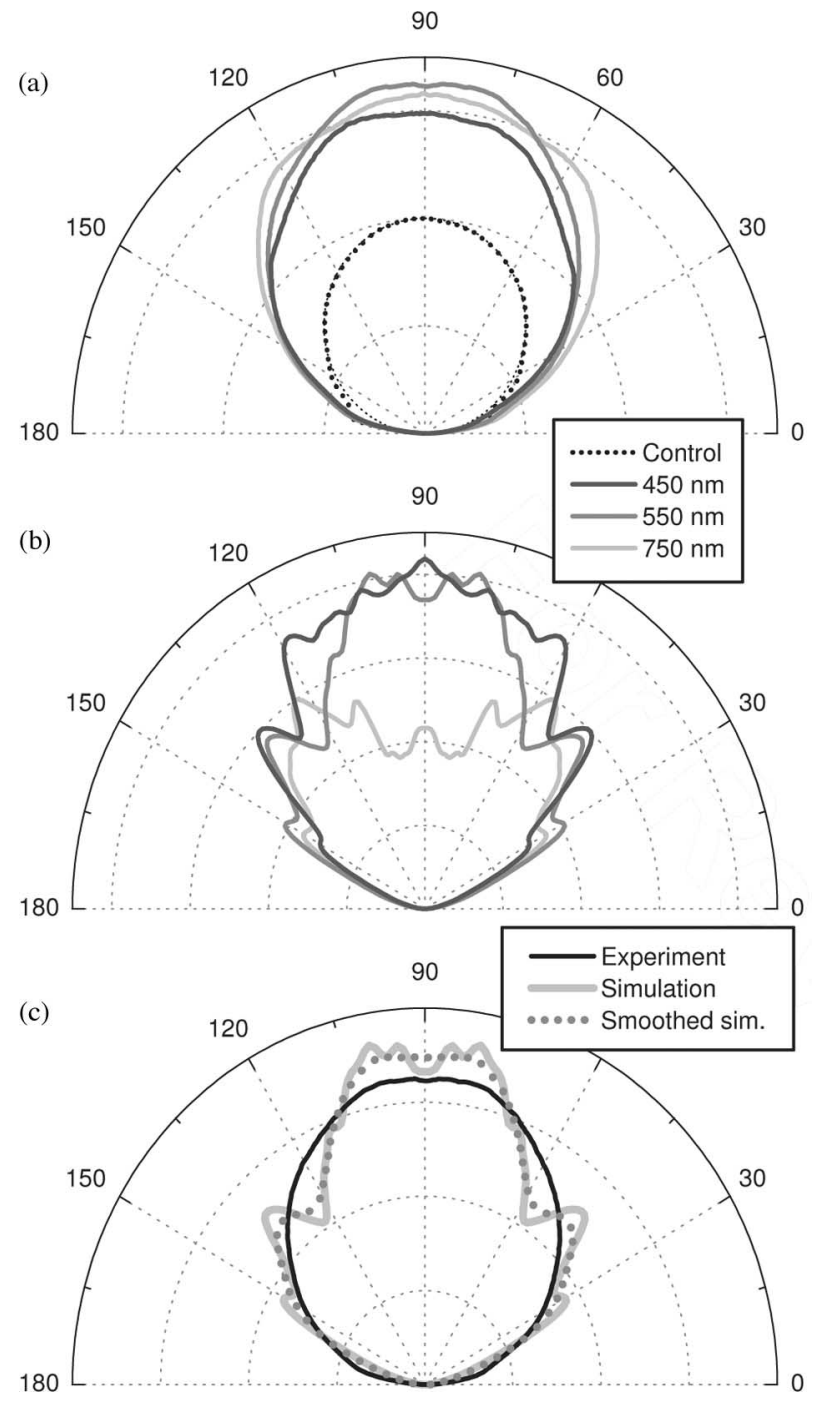

Fig. 4. (a) Measured far-field profile of the PCLEDs and control chip (unpolarized). (b) Simulated far-field profile of the PCLED (not to scale). (c) Qualitative comparison of the measured and simulated far-field profile for the $550 \mathrm{~nm}$ PCLED.

modulations that originate from interference between a complex set of criss-crossing Bragg scattered peaks. The line plots (bottom) correspond to horizontal slices in the contour plot at a single wavelength. These clearly reveal the 12 -fold symmetry of the quasi-crystal. Further, the intensity modulations are completely smeared out when the emission is integrated over the whole emission band. This is shown by the upper line plots for TE and TM polarization demonstrating there is negligible azimuthal dependence of the far-field profile. These results confirm the advantage of using PQCs over a more conventional hexagonal symmetry photonic crystal structure.

Integrating over a $\pm 90^{\circ}$ cone, the electroluminescence enhancement ranged between $42 \%-48 \%$ for all three pitches. However, integrating over a full $\pm 90^{\circ}$ cone masks a much larger enhancement over cones of smaller angle about the surface normal. The enhancement reached a maximum of $58 \%$ for a $\pm 15^{\circ}$ cone for the 550-nm-pitch PCLED, and $62 \%$ for a $\pm 45^{\circ}$ cone for the 750-nm-pitch PCLED.
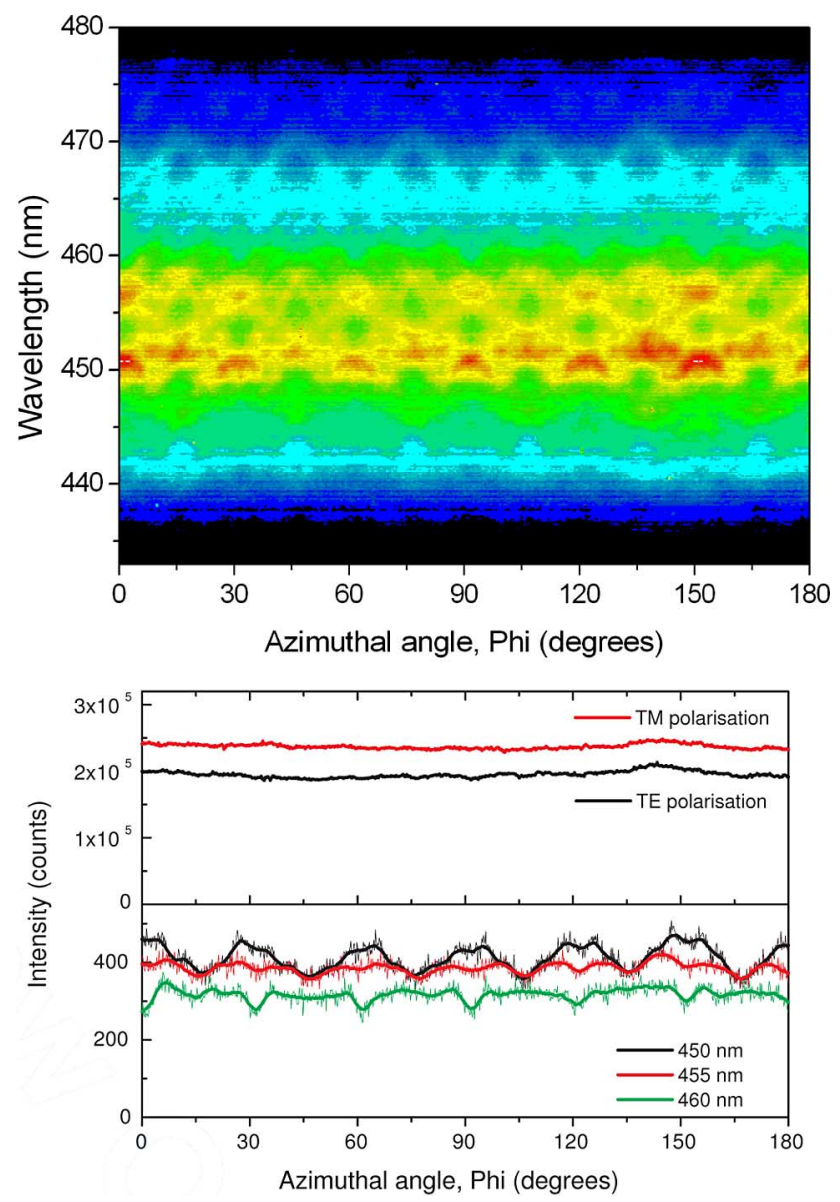

Fig. 5. Photoluminescence from a 730-nm-pitch PCLED for different azimuthal angles. The contour plot shows the intensity for a specific wavelength and azimuthal angle for TE polarized light. The lower line plots show the corresponding intensity at wavelengths of 450,455 , and $460 \mathrm{~nm}$, and highlight the 12 -fold symmetry of the PQC. However, once the spectra are integrated over all wavelengths (upper line plot), the 12 -fold symmetry is hardly visible.

The dependence on the photonic crystal hole size (i.e., air fraction) is shown in Fig. 6. For each pitch, increasing the hole size caused an increase in the total emission. This is most pronounced for the 550-nm-pitch PCLEDs. Since the hole size has no observable effect on the far-field distribution, the data in Fig. 6 can be summarized by integrating over a $\pm 90^{\circ}$ cone, and plotting the result against air fraction, as shown in Fig. 7.

Both the 450 and $550 \mathrm{~nm}$ pitches show similar increases in extraction from an air fraction from 0.13 to 0.21 . The $750 \mathrm{~nm}$ pitch also shows an increase up to 0.30 . Whilst the data does not point to a value for an optimum air fraction, it consistently shows that larger holes are better across a broad range of pitches. This result perhaps highlights the complex role of the etched layer in that it acts as both a 2-D photonic crystal laterally as well as an anti-reflection layer in the vertical direction. Further work is continuing to establish the optimum air fraction for the different pitches.

The optical properties of the PCLEDs have also been modeled using a 2-D finite-difference time domain (FDTD) technique with an in-line near- to far-field transform [16]. This allows the conversion of the near-field image of the light coming out of the 

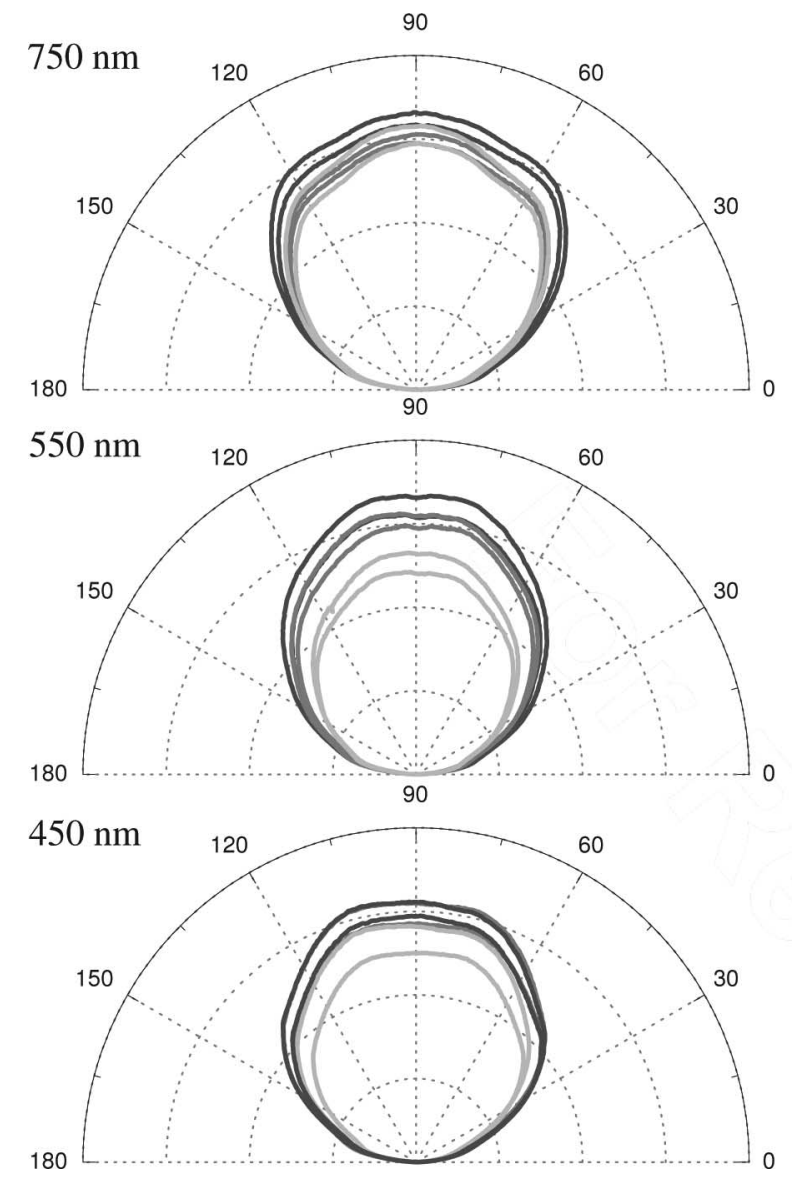

Fig. 6. Measured far-field emission profiles of PCLEDs with pitches of 450, 550 , and $750 \mathrm{~nm}$. The light gray, dark gray, and black lines correspond to the PCLEDs written with low, medium, and high electron beam doses.

LED into a far-field emission profile. The LED region is bound by reflecting boundaries in order to simulate a larger structure. A perfectly matched layer boundary condition is used elsewhere. Dipoles oriented to achieve TE-polarized light are placed in the quantum well region. The 2-D algorithm does not allow the full LED to be simulated. Therefore, only a cross section of the device is considered. The output of the simulations is then normalized to a Lambertian emitter in order to obtain far-field profiles.

Calculations of the extraction efficiency and far-field profile were carried out to inform the choice of pattern design. The $450 \mathrm{~nm}$ pitch was chosen for high on-axis emission whereas the $750 \mathrm{~nm}$ pitch was chosen for low on-axis and high off-axis emissions. Results of these calculations are shown in Fig. 4(b). Qualitatively, the simulations reproduce the key features in the measured enhanced extraction efficiency of the PCLEDs. In general, the maximum enhancement is at emission angles corresponding to the peak angles predicted from the simulations shown in Fig. 4(b).

Fig. 4(c) illustrates this further by comparing the simulated and measured angular dependence of the far-field intensity of the 550-nm-pitch PCLED. Note the simulations were carried out over a smaller spectral bandwidth as that of the measured data. Increasing the spectral range of the simulations will have the

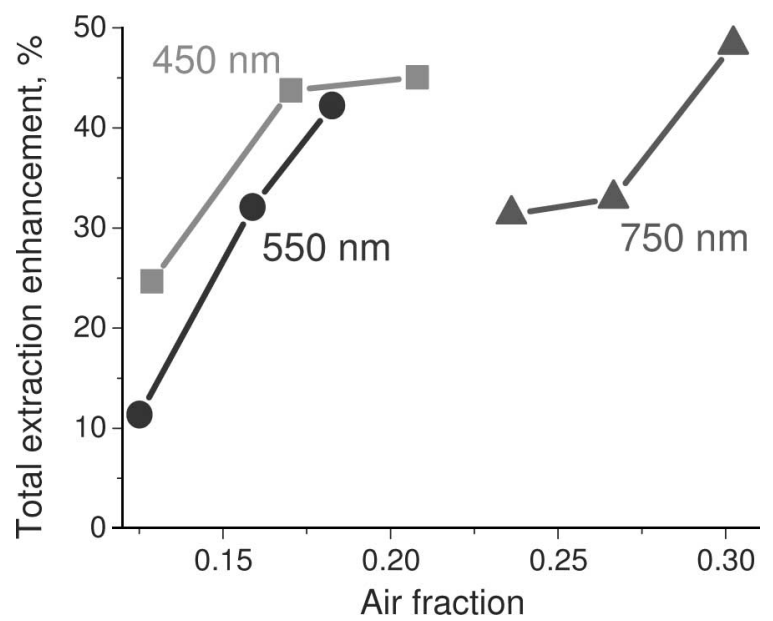

Fig. 7. Total extraction enhancement versus air fraction for the measured PCLEDs. A ratio was taken of the total intensity, integrated over a $\pm 90^{\circ}$ cone from the far-field profile, of patterned and unpatterned LEDs.

effect of smoothing the far-field pattern due to its slow variation with wavelength. Averaging the simulated data with respect to angle will have much the same effect. The dotted line in Fig. 4(c) shows the influence of smoothing on the simulated data. The degree of smoothing used here is arbitrary and nonoptimum. The shape of the averaged far-field pattern shows improved qualitative similarity with the experimental data. This demonstrates that the 2-D FDTD simulations are reproducing the key features in the experimental data, and are therefore a useful tool for optimizing photonic-quasi-crystal light-extraction elements.

\section{CONCLUSION}

In conclusion, results have been presented showing enhanced and more directional light extraction from blue-emitting LEDs with PQCs etched into the device surface. The results confirm a relatively smooth azimuthal dependence of the far-field profile once the broad emission band is taken into account, and point to a strong dependence on the extraction on the photonic crystal air fraction.

\section{ACKNOWLEDGMENT}

The authors would like to thank Dr. S. Yu and M. Redwood for the use of the ICP dry etching facility at the University of Bristol. D. W. E. Allsopp would like to thank the Royal Academy of Engineering and the Leverhulme Trust for providing a Senior Research Fellowship.

\section{REFERENCES}

[1] A. David, "High efficiency GaN-based LEDs: Light extraction by photonic crystals," Ann. de Physique, vol. 31, pp. 1-235, 2007.

[2] S. Fan, P. R. Villeneuve, and J. D. Joannopoulos, "High extraction efficiency of spontaneous emission from slabs of photonic crystals," Phys. Rev. Lett., vol. 78, pp. 3294-3297, 1997.

[3] J. J. Wierer, M. R. Krames, J. E. Epler, N. F. Gardner, M. G. Craford, J. R. Wendt, J. A. Simmons, and M. M. Sigalas, "InGaN/GaN quantumwell heterostructure light-emitting diodes employing photonic crystal structures," Appl. Phys. Lett., vol. 84, pp. 3885-3887, 2004. 
[4] K. Orita, S. Tamura, T. Takizawa, T. Ueda, M. Yuri, S. Takigawa, and D. Ueda, "High-extraction-efficiency blue light-emitting diode using extended-pitch photonic crystal," Jpn. J. Appl. Phys., vol. 43, pp. 58095813, 2004.

[5] T. N. Oder, J. Shakya, J. Y. Lin, and H. X. Jiang, "III-nitride photonic crystals," Appl. Phys. Lett., vol. 83, pp. 1231-1233, 2003.

[6] P. M. Pattison, A. David, R. Sharma, C. Weisbuch, S. Denbaars, and S. Nakamura, "Gallium nitride based microcavity light emitting diodes with $2 \lambda$ effective cavity thickness," Appl. Phys. Lett., vol. 90, pp. 0311111-031111-3, 2007.

[7] A. David, B. Moran, K. McGroddy, E. Matioli, E. L. Hu, S. P. DenBaars, S. Nakamura, and C. Weisbuch, "GaN/InGaN light emitting diodes with embedded photonic crystal obtained by lateral epitaxial overgrowth," Appl. Phys. Lett., vol. 92, pp. 113514-1-113514-3, 2008.

[8] M.-K. Kwon, J.-Y. Kim, I.-K. Park, K.-S. Kim, G.-Y. Jung, S.-J. Park, J. W. Kim, and Y. C. Kim, "Enhanced emission efficiency of GaN/InGaN multiple quantum well light-emitting diode with an embedded photonic crystal," Appl. Phys. Lett., vol. 92, pp. 251110-1-251110-3, 2008.

[9] C.-F. Lai, H. C. Cho, C. H. Chao, H. T. Hsueh, J.-F. T. Wang, W. Y. Yeh, and J. Y. Chi, "Anisotropy of light extraction from two-dimensional photonic crystal light-emitting diodes," Appl. Phys. Lett., vol. 91, pp. 123117123117-3, 2007

[10] M. E. Zoorob, M. D. B. Charlton, G. J. Parker, J. J. Baumberg, and M. C. Netti, "Complete photonic bandgaps in 12-fold symmetric quasicrystals," Nature, vol. 404, pp. 740-743, 2000.

[11] A. David, T. Fujii, E. Matioli, R. Sharma, S. Nakamura, S. P. DenBaars, C. Weisbuch, and H. Benisty, "GaN light-emitting diodes with Archimedean lattice photonic crystals," Appl. Phys. Lett., vol. 88, pp. 073510-1-073510-3, 2006.

[12] Z. S. Zhang, B. Zhang, J. Xu, K. Xu, Z. J. Yang, Z. X. Qin, T. J. Yu, and D. P. Yu, "Effects of symmetry of GaN-based two-dimensional photonic crystal with quasicrystal lattices on enhancement of surface light extraction," Appl. Phys. Lett., vol. 88, pp. 171103-1-171103-3, 2006.

[13] D. Englund and J. Vučković, "A direct analysis of photonic nanostructures," Opt. Exp., vol. 14, pp. 3472-3483, 2006.

[14] M. F. Schubert, S. Chhajed, J. K. Kim, E. F. Schubert, and J. Cho, "Polarization of light emission by $460 \mathrm{~nm}$ GaInN/GaN light-emitting diodes

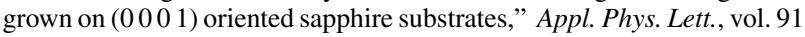
pp. 051117-1-051117-3, 2007.

[15] M. C. Netti, A. Harris, J. J. Baumberg, D. M. Whittaker, M. D. B. Charlton, M. E. Zoorob, and G. J. Parker, "Optical trirefringence in photonic crystal waveguides,” Phys. Rev. Lett., vol. 86, no. 8, pp. 1526-1529, 2001.

[16] A. Taflove and S. C. Hagness, Computational Electrodynamics: The Finite-Difference Time Domain Method, 3rd ed. Norwood, MA: Artec House, 2005.

Philip A. Shields received the M.Phys. and D.Phil. degrees from the Department of Physics, University of Oxford, Oxford, U.K., 1997 and 2001, respectively.

From 2001 to 2004, he was with the University of Oxford to run a metalorganic vapor phase density (MOVPE) crystal growth reactor for arsenide- and antimonide-based semiconductors. Since 2004, he has been with the University of Bath, Bath, U.K., where he has been engaged in the application of nitride-based semiconductors for optoelectronics applications. He is currently a Research Fellow and is involved in laser diode and LED device processing. His current research interests include novel device designs.

Martin D. B. Charlton received the B.Sc. degree in physics and electronics and the M.Sc. degree from the University of Reading, Reading, U.K., in 1991 and 1994, respectively, and the Ph.D. degree from the University of Southampton, Southampton, U.K., in 1999.

He was the founder of a start-up company, Mesophotonics Ltd., Southampton through which he commercialized work on photonic crystal devices, surfaceenhanced Raman scattering (SERS) sensors, and high-brightness LEDs. He is currently with the University of Southampton, where he currently holds a Royal Society Research Fellowship at the School of Electronics and Computer Science. He is currently engaged in photonic crystals super-continuum lasers, biosensing, and solid-state light sources.

Dr. Charlton received the "Rank Thesis" Prize in 1999 for his Ph.D. degree on fabrication and modeling of planar photonic crystal devices.
Tom Lee received the B.Eng. and Ph.D. degrees from the Department of Electronics and Computer Science, University of Southampton, Southampton, U.K., in 2002 and 2006, respectively.

From 2006 to 2008, he was with Mesophotonics Ltd., where he was engaged in the design and modeling of photonic crystal LED devices. Since 2008, he has been a Senior Engineer at PhotonStar LED Ltd., Southampton.

Majd E. Zoorob received the B.Eng. and Ph.D degrees from the Department of Electronics and Computer Science, University of Southampton, Southampton, U.K., in 1997 and 2000, respectively.

From 2001 to 2007, he was a Co-Founder of Mesophotonics Ltd., and was engaged in the design and modeling of photonic crystal devices. Since 2007, he has been the Chief Technology Officer (CTO) and Co-Founder of PhotonStar LED Ltd., Southampton.

Duncan W. E. Allsopp (M'89) received the B.Sc. degree in physics, and the M.Sc. and Ph.D. degrees from the University of Sheffield, Sheffield, U.K., in 1971, 1974, and 1977, respectively.

From 1977 to 1979, he was with Ferranti Electronics Ltd., where he was engaged in developing high-speed Si bipolar transistors. From 1979 to 1984 , he was with the Institute of Science and Technology, University of Manchester, Manchester, U.K., where he was involved in the research on defects in semiconductors. From 1984 to 1986, he was with British Telecom Research Laboratories, Martlesham Heath, U.K. In 1986, he joined the University of York, York, U.K., where he established a Group researching on photonic devices. Since 1999, he has been with the Optoelectronics Group, University of Bath, Bath, U.K., where he is currently a Royal Academy of Engineering/Leverhulme Trust Senior Research Fellow, where he continues his research in photonics.

Wang N. Wang (M'89) received the Ph.D. degree in physical chemistry from Cambridge University, Cambridge, U.K. in 1988.

In March 1999, he was the Industrial Chair in the Department of Physics, University of Bath, Bath, U.K., where he has been with the Department of Electronic and Electrical Engineering since March 2007. He was engaged in generating significant IP relating to GaN-based materials, LEDs, and laser diodes. He holds 31 patents filed, 18 granted in U.K., U.S.A., Japan, Taiwan, and China. His current research interests include the growth and microstructure characterization of III-V nitride materials by metal-organic chemical vapor deposition (MOCVD) and hydride vapor phase epitaxy (HVPE), design, modeling, and fabrication of optoelectronic devices based on III-V nitride compound semiconductors. 